\title{
Research Article \\ Extremely elevated erythrocyte sedimentation rate: patient diagnoses at a tertiary Indian hospital
}

\author{
Christol Blanch Moras ${ }^{1}$, Ashima Nagesh Amin², Reshma Gopal Kini ${ }^{3}$ \\ ${ }^{1,2}$ Assistant Professor, ${ }^{3}$ Associate Professor, Department of Pathology, Father Muller Medical College Mangalore, Karnataka, \\ India
}

(Received: October $2020 \quad$ Revised: November $2020 \quad$ Accepted: December 2020)

Corresponding author: Christol Blanch Moras. Email: christol.b.moras@gmail.com

\begin{abstract}
Introduction and Aim: Extremely elevated Erythrocyte Sedimentation Rate (EEESR) is the term commonly used for elevation of ESR more than or equal to $100 \mathrm{~mm}$ in the first hour. To the best of our knowledge, this is the first study in Indian population. Aim was to study the common etiologies of EEESR in Indian patients at a tertiary hospital.

Materials and Methods: A retrospective, cross- sectional and descriptive study was carried out and included all patients 18 years of age and above, and who had a raised ESR of $100 \mathrm{~mm}$ and above at the end of $1^{\text {st }}$ hour at least one time between January 2018 to December 2018 and tested at our institute. The patient's demographic details, ESR values and diagnoses were retrieved from electronic records and were divided into 7 categories like infections, malignancy, renal diseases, autoimmune diseases, mixed diagnoses, miscellaneous and unknown.

Results: During the study period, 607 patients had EEESR. There were 335 males and 272 females. The mean age was 55 years. Within the broad categories of diseases, infection was the most common cause (52\%) followed in descending order by renal diseases (12.4\%), malignancy (9.6\%), miscellaneous (8.4\%), mixed diagnoses $(7.6 \%)$, autoimmune diseases (7\%) and unknown (3\%). In the infection category, pneumonia was the commonest.

Conclusion: This study on frequency of common etiologies of EEESR is the first study in Indian population. Our study reaffirms findings that most patients have an underlying cause for EEESR. The commonest etiology in Indian patients is infection with pneumonia being the frequent cause.
\end{abstract}

Keywords: Infection; pneumonia; renal diseases; malignancy

\section{INTRODUCTION}

E SR (Erythrocyte sedimentation rate) is an inexpensive and a simple test most widely used in clinical practice. It measures the rate at which the red blood cells sediment in a period of time and is expressed in terms of $\mathrm{mm}$. Being a surrogate marker of acute phase reaction, it has been proposed by some authors to use as sickness index $(1,2)$. The test is best used as a tool to follow the clinical course in diseases like Lymphomas, Rheumatoid Arthritis, Temporal arteritis and Polymyalgia Rheumatica (3).

Extremely elevated ESR (EEESR) is the term commonly used for elevation of ESR more than or equal to $100 \mathrm{~mm}$ in the first hour. EEESR has numerous etiologies and is commonly associated with an underlying disease posing a diagnostic challenge to the treating clinician. EEESR is found in several diseases including malignancies, infection and collagen vascular diseases $(4,5)$. Currently, there is no data on the frequency of diseases with EEESR in Indian population. This study was carried out to address the frequency of common etiologies of EEESR in a south Indian tertiary hospital.

\section{MATERIALS AND METHODS}

A retrospective, cross- sectional and descriptive study was carried out and included all patients 18 years of age and above, and who had a raised ESR of $100 \mathrm{~mm}$ and above at the end of $1^{\text {st }}$ hour at least one time between January 2018 to December 2018 and tested at our institute. The study was approved by the ethical committee. The ESR at this hospital is carried out on whole blood samples obtained through standard venous veni-puncture techniques in black top vacutainer containing $3.8 \%$ sodium citrate anticoagulant ( $1.6 \mathrm{ml}$ blood in $0.4 \mathrm{ml}$ anticoagulant). The analysis is done manually by Westergren method using Westergren pipette. Results are expressed as the fall in RBCs in $\mathrm{mm}$ at the end of first hour.

Inclusion criteria were all patients of 18 years of age and above, and who had a raised ESR of $100 \mathrm{~mm}$ and above at the end of $1^{\text {st }}$ hour at least one time.

The patients' demographic details namely age, sex along with clinical diagnosis and ESR value were retrieved from electronic medical records. As in previous studies, the patients were divided into 7 categories like infections, malignancy, renal diseases, autoimmune diseases, mixed diagnoses, miscellaneous and unknown (6-8).

Those patients who had more than 1 clinical diagnosis and which was known in the literature to be associated with elevated ESR were categorised as mixed diagnoses. When disease name was not specified, the diagnosis was categorised as unknown. 
The data were computed using Microsoft excel 2010 and by descriptive statistics the total numbers, mean value and percentage of different groups were calculated. The aim was to study the common etiologies of EEESR in Indian patients at a tertiary hospital.

\section{RESULTS}

Table 1: Etiology of extremely elevated ESR with a gender distribution

\begin{tabular}{|c|c|c|c|c|c|c|c|}
\hline \multirow{2}{*}{ Etiology } & \multicolumn{2}{|c|}{ Male } & \multicolumn{2}{c|}{ Female } & \multicolumn{2}{c|}{ Total } & \multirow{2}{*}{$\begin{array}{c}\text { Mean age of } \\
\text { all patients }\end{array}$} \\
\cline { 2 - 7 } & $\mathrm{N}$ & $\%$ & $\mathrm{~N}$ & $\%$ & $\mathrm{n}$ & $\%$ & 55 \\
\hline Infections & 175 & 28.8 & 140 & 23.2 & 315 & 52 & 59 \\
\hline Renal & 45 & 7.4 & 30 & 5 & 75 & 12.4 & 58 \\
\hline Malignancy & 28 & 4.6 & 30 & 5 & 58 & 9.6 & 55 \\
\hline Miscellaneous & 30 & 4.9 & 21 & 3.5 & 51 & 8.4 & 55 \\
\hline Mixed & 30 & 4.9 & 16 & 2.7 & 46 & 7.6 & 44 \\
\hline Autoimmune & 14 & 2.3 & 29 & 4.7 & 43 & 7 & 60 \\
\hline Unknown & 13 & 2.1 & 6 & 0.9 & 19 & 3 & 55 \\
\hline Total & 335 & 55 & 272 & 45 & 607 & 100 & \\
\hline
\end{tabular}

Within the broad categories of diseases, infection was the most common cause followed in descending order by renal diseases, malignancy, miscellaneous, mixed diagnoses, autoimmune diseases and unknown as summarised in the table 1.

Among the types of infection, the commonest was pneumonia followed by infected ulcers and urinary tract infections as shown in table 2 .

Table 2: Extremely elevated ESR due to infections $(\mathrm{n}=315)$

\begin{tabular}{|c|c|c|}
\hline Type of infection & $\mathbf{n}$ & $\%$ \\
\hline Pneumonia & 73 & 23.17 \\
\hline Infected ulcers & 61 & 19.36 \\
\hline Urinary tract infections & 42 & 13.3 \\
\hline Sepsis & 33 & 10.47 \\
\hline Osteomyelitis & 28 & 8.8 \\
\hline Tuberculosis & 24 & 7.6 \\
\hline Abscess & 18 & 5.7 \\
\hline Cellulitis & 10 & 3.17 \\
\hline Leptospirosis & 9 & 2.95 \\
\hline Septic arthritis & 8 & 2.5 \\
\hline Other infections & 9 & 2.95 \\
\hline
\end{tabular}

Other infections in 9 patients included 3 cases of HIV infection, 3 cases of Hansen's disease with one each case of Malaria, Ricketsia and Dengue.

The second most common diagnostic category were renal diseases. Among the types of renal diseases, chronic kidney disease was the most common as shown in table 3 .

The third most common diagnostic category was malignancy. Non haematological malignancies namely carcinoma of solid organs was more common than haematological malignancies as summarised in table 4.

The fourth most common diagnostic category were the miscellaneous causes. In this category, traumatic causes were the commonest as shown in table 5 .
During the study period, 607 patients had EEESR. There were 335 males and 272 females. The mean age was 55 years. The mean EEESR was $120 \mathrm{~mm} / \mathrm{hr}$, highest being $180 \mathrm{~mm} / \mathrm{hr}$ and was seen in adenocarcinoma stomach with metastatic lymph nodes.
Table 3: Extremely elevated ESR due to renal causes $(\mathrm{n}=75)$

\begin{tabular}{|c|c|c|}
\hline Renal causes & n & \% \\
\hline Chronic kidney disease & 40 & 53.3 \\
\hline Acute kidney injury & 17 & 22.7 \\
\hline $\begin{array}{c}\text { Acute kidney injury on chronic } \\
\text { kidney disease }\end{array}$ & 14 & 18.7 \\
\hline Glomerulonephritis & 4 & 5.3 \\
\hline
\end{tabular}

Table 4: Extremely elevated ESR due to malignancy $(\mathrm{n}=58)$

\begin{tabular}{|c|c|c|}
\hline Malignancies & n & \% \\
\hline Multiple myeloma & 9 & 15.5 \\
\hline Leukemias * & 6 & 10.3 \\
\hline MDS & 1 & 1.7 \\
\hline Carcinoma lung & 5 & 8.6 \\
\hline Carcinoma ovary & 5 & 8.6 \\
\hline Others \# & 32 & 55.2 \\
\hline
\end{tabular}

*Out of 6 cases of leukemias, 3 were acute myeloid leukemia, 1 each were chronic lymphocytic leukemia, acute lymphoblastic leukemia and chronic myelomonocytic leukemia.

\#out of the 32 other cancers, 3 each were carcinoma colon, carcinoma breast, carcinoma stomach, carcinoma oesophagus, carcinoma cervix, carcinoma buccal mucosa.

2 each were carcinoma endometrium, carcinoma hypopharynx, 1 each were leiomyosarcoma uterus, carcinoma glottis, carcinoma prostate, carcinoma pancreas, carcinoma tongue, periampullary carcinoma, spindle cell sarcoma, carcinoma bladder, renal cell carcinoma, metastatic squamous cell carcinoma with unknown primary. 
Table 5: Extremely elevated ESR due to miscellaneous causes $(n=51)$

\begin{tabular}{|c|c|c|}
\hline Miscellaneous causes & $\mathbf{n}$ & $\mathbf{\%}$ \\
\hline Traumatic & 16 & 31.3 \\
\hline Liver diseases & 10 & 19.6 \\
\hline Venous thromboembolism & 5 & 9.8 \\
\hline Ischemic heart disease & 5 & 9.8 \\
\hline Severe anemia & 4 & 7.9 \\
\hline others & 11 & 21.5 \\
\hline
\end{tabular}

Others include 3 each of pregnancy, endometriotic cyst, 2 each of heart failure, interstitial lung disease, 1 of acute ischemic stroke.

Table 6: Extremely elevated ESR due to autoimmune causes(n=43)

\begin{tabular}{|c|c|c|}
\hline Autoimmune diseases & n & \% \\
\hline Rheumatoid arthritis & 21 & 48.8 \\
\hline Systemic lupus erythematosus & 12 & 27.9 \\
\hline Psoriasis & 5 & 11.6 \\
\hline others & 5 & 11.6 \\
\hline
\end{tabular}

Others include 1 each of mixed connective tissue disease, vasculitis, autoimmune hepatitis, granulomatosis with polyangiitis, undifferentiated connective tissue disease.

The fifth most common diagnostic category in our study were mixed diagnoses. The EEESR in this category of patients could not be attributed to a single cause.

In this category there were 6 each of chronic kidney disease with pneumonia, chronic kidney disease with infected ulcers; 4 each of chronic kidney disease with rheumatoid arthritis, acute kidney injury with ulcer, 3 each of chronic kidney disease with pyelonephritis, urinary tract infections with pneumonia, Acute kidney injury with osteomyelitis; 2 each of chronic kidney disease with Tuberculosis, Chronic kidney disease with liver disease and ischemic heart disease, liver disease with pneumonia, fracture with chronic kidney disease, 1 each of tuberculosis with HIV infection, chronic kidney disease with metastatic lymph node with unknown primary.

The sixth most common diagnostic category was autoimmune diseases, with rheumatoid arthritis being the commonest in this category as shown in table 6 . Unknown causes comprised 19\% of all cases.

\section{DISCUSSION}

EEESR is a sensitive indicator of disease and is associated with a positive predictive value of $90 \%$ when correlating with certain diagnosis (9). To our knowledge this is the first study on frequency of diseases with EEESR in Indian population.

In an initial study done by Zacharski et al., found that malignancy was the commonest etiology for EEESR constituting $58 \%$ of cases (10). In another study done by Stein and Xavier found inflammatory diseases as the commonest cause constituting $71 \%$ of the cases (11). In another study done by Ohta et al., on Japanese patients found inflammatory disease namely pseudogout as the commonest etiology for EEESR (4). However various studies have found infection as the commonest cause for EEESR followed by collagen vascular diseases and malignancy $(6,7)$.

Similar to previous studies, we found $97 \%$ of patients had a known clinical diagnosis indicating that a severe disease is usually associated with EEESR and that infection was the commonest etiology $(6,7)$. In the study done by Yousuf et al., on Saudi patients reported infection as the commonest etiology with osteomyelitis being the frequent cause (6).

Compared to our study, data from patients examined at Mayo Clinic, Minnesota too detected infection as the commonest cause with pneumonia being common in this category. Our study too had $23.17 \%$ of pneumonia. The possible explanation could be pneumonia being commonest cause of morbidity and mortality in Indian population with increased incidence in elderly adults. Aging affects immune function and thereby making the older individuals susceptible for infection. This finding is also related to increased rates of smoking and alcoholism together with cumulative effects of co-morbid chronic diseases like COPD, Diabetes mellitus, Hypertension and their long-term treatment seen in these patients (12). In addition, advanced diagnostic and therapeutic protocols have led to increase in prevalence of these chronic diseases along with emergence of multidrug resistant organisms which poses an increased risk in these patients $(7,12)$.

The other main infections in our study were infected wounds and urinary tract infections. Most of the infected wounds comprised of infected skin ulcers in diabetic patients. This could be attributed to the fact that patients with Diabetes mellitus are prone to severe foot infections due to neuropathy and vascular dysfunction (13). Supporting this concept is previous research done by Hadavand et al., indicating that the ESR in patients with infected ulcers was significantly higher than that of patients with non-infected diabetic ulcers (13).

Renal diseases were second most common cause for EEESR in our study. This result is unlike previous studies where renal diseases were either fourth or fifth cause for EEESR. This result could be attributed to the fact that our hospital being a tertiary referral centre has a nephrology unit which caters to patients with renal diseases. The most common renal associated diagnoses in our study were chronic kidney disease, acute kidney injury, acute kidney injury on chronic kidney disease which is similar to previous studies. These diseases are frequent causes for EEESR and therefore ESR may not always serve as an indicator of infections which is frequently seen in these patients $(5,14)$. A study done by Al-Homrany indicated that 
fibrinogen was the only factor which correlated significantly and independently with ESR. Compared to healthy controls, uremic patients had significant increase in quantity and activity of fibrinogen (5).

The third leading cause for EEESR in our study was malignancy. Non haematological malignancy being more common comprising $72.4 \%$ of all malignancies. This is different from the results previously reported. In a study by Yousuf et al., done on Saudi patients, haematological malignancy namely lymphoma, multiple myeloma and leukemia were the most common malignancies associated with EEESR (6).

In another study done by Haque et al., reported haematological malignancies as the only cause for EEESR in this category (3).

Our results indicated that carcinoma of solid organs was frequent etiology for EEESR with Carcinoma lung and carcinoma ovary being more common. An ESR of 100 and above usually indicates metastasis in patients with solid tumors. The high ESR in these patients has a poor prognosis $(15,16)$. ESR however should not be used as a screening test for malignancy as the sensitivity of an EESR for malignancy is low $(9,11,14)$.

Among haematological malignancies, myeloma comprised $15.5 \%$ of all the cases. Myeloma is known to have a strong association with raised ESR wherein clonal proliferation of plasma cells secrete immunoglobulins leading to plasma hyper viscosity (17).

However, unlike previous studies, our study failed to identify even a single cause of lymphoma for EEESR in this category. EEESR in Hodgkin's Lymphoma is a predictor of early relapse. The rates of elevated values of ESR reflect the course of disease and response to treatment in these patients (18).

Our study had $8.4 \%$ of miscellaneous causes for EEESR. Commonest etiology in this category was trauma induced by accidents, fractures and surgical operations. In a study done by Garnavos et al., revealed that intramedullary nailing following fractures causes significant inflammatory reaction thereby causing sharp rise in ESR postoperatively. ESR often increases to high levels after major surgeries or extensive trauma and usually returns to normal within 6 months (19). Liver diseases were second most common in this group. The possible explanation for this is alcoholic liver cirrhosis being the commonest cause of chronic liver disease in India $(20,21)$. Chronic alcohol consumption is associated with inflammation. Alcohol has adverse effect on haematopoiesis contributing to macrocytosis and anemia thereby causing further increase in ESR (20, 21).

Our study had $7.6 \%$ of mixed diagnoses. These patients had more than 1 clinical diagnosis which was known in the literature to be associated with elevated ESR. The association of chronic kidney disease with infection was more common in this category. This supports the concept of previous studies which report that infection is often co-existent with other diseases in patients with EEESR and that multimorbidity is a risk factor $(6,7)$. One of the patients in our study who had chronic kidney disease was diagnosed with metastatic lymph node of unknown primary and therefore malignancy can be easily overlooked in this rare occurrence.

Autoimmune diseases attributed to $7 \%$ of cases. The commonest etiology in this category being rheumatoid arthritis. This is in agreement with other similar studies. The EEESR in these patients indicates a state of heightened systemic inflammation and disease flare. EEESR may also signal heart failure in these patients (22). Second commonest etiology was systemic lupus erythematosus (SLE). In a study done by Firooz et al., ESR levels did not differ significantly between patients with disease flare and active infection (23).

\section{Strength of the study}

To our knowledge this is the first study where we have tried to identify the causes of EEESR in Indian population in a tertiary care setting.

\section{CONCLUSION}

Our study reaffirms findings indicating that most patients have an underlying cause for EEESR and that infection is the commonest etiology in Indian patients. Mere finding of EEESR should not trigger expensive investigations and that widely available tests along with meticulous physical examination will help in reaching a diagnosis.

\section{CONFLICT OF INTEREST}

Authors declare no conflict of interest.

\section{REFERENCES}

1. Brigden, M. L. Clinical Utility of the Erythrocyte Sedimentation Rate. Am Fam Physician. 1999;60(5):14431450.

2. Haque, M. D. Z., Alam, S. M. Z., Noman, M., Siddique, M. A. N., Azhar, M. A. laboratory tests required to reach the diagnosis in patients with grossly elevated ESR. J Medicine. 2009; 10: 7-11.

3. Laboratory Haematology. Part 1 Wintrobe's Clinical Haematology $13^{\text {th }}$ edition. Pages $16-17$.

4. Ohta, R., Kaneko, M., Hattori, S. Diseases with Extremely elevated ESR in secondary healthcare facility: Retrospective cohort study. Shimane journal of medical sciences. Jan 2017; 34:27-33.

5. Al-Homrany, M. The Significance of Extreme Elevation of the Erythrocyte Sedimentation Rate in Hemodialysis Patients. Saudi J Kidney Dis Transplant. 2002; 13(2): 141-145.

6. Yousuf, M., Akhter, J., Khairy, K. A., Al-Saadan, M.A., Salih Bin- Salih. Extremely Elevated Erythrocyte Sedimentation Rate etiology at a tertiary care center in Saudi Arabia. Saudi Med J. 2010; 31(1): 1227-1231.

7. Daniels, L. M., Tosh, P. K., Fiala, J. A., Schleck, C. D., Mandrekar, J. N., Beckman, T. J. Extremely Elevated 
Erythrocyte Sedimentation Rates: Associations with Patients' Diagnoses, Demographic Characteristics, and Comorbidities. Mayo Clin Proc. 2017; 92(11): 1636-1643.

8. Levay, P. F., Retief, J. H. Causes of high erythrocyte sedimentation rates in an inpatient population. SAMJ. January 2005; 95(1): 45-46.

9. Fincher, R.M.E., Page, M. I. Clinical Significance of Extreme Elevation of the Erythrocyte Sedimentation Rate. Arch Intern Med. 1986; 146(8): 1581-1583.

10. Zacharski, L. R., Kyle, R. A. Significance of extreme elevation of ESR rate. The Journal of the American Medical Association. 1967; 202(4): 264-266.

11. Stein, C. M., Xavier, R. Extreme Elevation of the Erythrocyte Sedimentation Rate in Patients Admitted to a General Medical Ward in Harare, Zimbabwe. Jou Tro Med and Hyg. 1989; 92(4): 259-62.

12. Abdullah, B. B., Zoheb, M., Ashraf, S. M., Ali, S., Nausheen, N. A Study of Community-Acquired Pneumonias in Elderly Individuals in Bijapur, India International Scholarly Research Network ISRN Pulmonology. 2012, Article ID 936790.

13. Amouzeg, F.H.A., Amid, H. Pro-Calcitonin, Erythrocyte Sedimentation Rate and C - reactive Protein in Predicting Diabetic Foot Ulcer Characteristics; a Cross Sectional Study. Archives of Academic Emergency Medicine. 2019; 7 (1): e37.

14. Abbag, F. I., Qehtani, J. M. A. Extreme elevation of ESR in children. Ann Saudi Med. 2007; 27(3): 175-178.

15. Tas, F., Erturk. K. Elevated ESR is associated with metastatic disease and worse survival in patients with cutaneous malignant melanoma. Molecular and clinical oncology. 2017; 7: 1142-1146.

16. Watson, J., Salisbury, C., Banks, J., Whiting, P., Hamilton, W. Predictive value of inflammatory markers for cancer diagnosis in primary care: a prospective cohort study using electronic health records. British Journal of Cancer. 2019; 120: 1045-1051.

17. Koshiaris, C., Bruel, A.V.D., Oke, J. L., Nicholson, B. D., Shephard, E., Braddick, M., Hamilton, W. Early detection of multiple myeloma in primary care using blood tests: a casecontrol study in primary care. British Journal of General Practice, September. 2018; e586-e593.

18. Bien, E., Balcerska, A. Serum Soluble Interleukin-2 Receptor, Beta2-Microglobulin, Lactate Dehydrogenase and Erythrocyte Sedimentation Rate in Children with Hodgkin's Lymphoma. Scandinavian Journal of Immunology. 2009; 70: 490-500.

19. Garnavos, C., Xirou, S. T., Nikolatos, A., Kanakaris, N., Tzortzi, P., Balbouzis, T., Papavasiliou, N. Alteration of Body Temperature, Erythrocyte Sedimentation Rate, and Creactive Protein After Reamed Intramedullary Nailing A Prospective Study. J Orthop Trauma. 2005; 19: 323-328.

20. Patil, A. M., Arifulla, M. K., Yendigeri, S. M., Sajanar, B. B. Study of Alcoholic Liver Cirrhosis in Hospital Based Patients, Bijapur, Northern Karnataka, India. International Journal of Current Medical and Applied Sciences. June 2015; 7(1): 16-20.

21. Mukherjee, P. S., Vishnubhatla, S., Amarapurkar, D. N., Das, K., Sood, A., Chawla,Y. K., Eapen, C. E., et al., Etiology and mode of presentation of chronic liver diseases in India: A multi centric study. PLoS ONE 12(10): e0187033.

22. Kremers, H. M., Nicola, P. J., Crowson, C. S., Ballman, K. V., Jacobsen, S. J., Roger, V. L., Gabriel, S. E. Raised erythrocyte sedimentation rate signals heart failure in patients with rheumatoid arthritis. Ann Rheum Dis 2007; 66: 76-80.

23. Firooz, N., Albert, D. A., Wallace, D. J., Ishimori, M., Berel, D., Weisman,M. H. High-sensitivity C-reactive protein and erythrocyte sedimentation rate in systemic lupus erythematosus. LUPUS. May 2011; 20(6): 588-597. 